\title{
Neural Decoding of Bistable Sounds Reveals an Effect of Intention on Perceptual Organization
}

\author{
-Alexander J. Billig, Matthew H. Davis, and @Robert P. Carlyon \\ Medical Research Council Cognition and Brain Sciences Unit, University of Cambridge, Cambridge CB2 7EF, United Kingdom
}

Auditory signals arrive at the ear as a mixture that the brain must decompose into distinct sources based to a large extent on acoustic properties of the sounds. An important question concerns whether listeners have voluntary control over how many sources they perceive. This has been studied using pure high $(\mathrm{H})$ and low $(\mathrm{L})$ tones presented in the repeating pattern HLH-HLH-, which can form a bistable percept heard either as an integrated whole (HLH-) or as segregated into high (H-H-) and low (-L-) sequences. Although instructing listeners to try to integrate or segregate sounds affects reports of what they hear, this could reflect a response bias rather than a perceptual effect. We had human listeners ( 15 males, 12 females) continuously report their perception of such sequences and recorded neural activity using MEG. During neutral listening, a classifier trained on patterns of neural activity distinguished between periods of integrated and segregated perception. In other conditions, participants tried to influence their perception by allocating attention either to the whole sequence or to a subset of the sounds. They reported hearing the desired percept for a greater proportion of time than when listening neutrally. Critically, neural activity supported these reports; stimulus-locked brain responses in auditory cortex were more likely to resemble the signature of segregation when participants tried to hear segregation than when attempting to perceive integration. These results indicate that listeners can influence how many sound sources they perceive, as reflected in neural responses that track both the input and its perceptual organization.

Key words: auditory streaming; intention; $\mathrm{MEG;} \mathrm{multivariate} \mathrm{classification;} \mathrm{perceptual} \mathrm{organization;} \mathrm{stream} \mathrm{segregation}$

\section{Significance Statement}

Can we consciously influence our perception of the external world? We address this question using sound sequences that can be heard either as coming from a single source or as two distinct auditory streams. Listeners reported spontaneous changes in their perception between these two interpretations while we recorded neural activity to identify signatures of such integration and segregation. They also indicated that they could, to some extent, choose between these alternatives. This claim was supported by corresponding changes in responses in auditory cortex. By linking neural and behavioral correlates of perception, we demonstrate that the number of objects that we perceive can depend not only on the physical attributes of our environment, but also on how we intend to experience it.

\section{Introduction}

For us to make sense of our environment, the brain must determine which elements of energy arriving at the sensory organs arise from the same source and should therefore be perceptually grouped. In audition, the less rapidly that sequential sounds change in one or more physical quantities, such as frequency,

\footnotetext{
Received Oct. 19, 2017; revised Jan. 21, 2018; accepted Feb. 6, 2018.

Author contributions: A.J.B., M.H.D., and R.P.C. designed research; A.J.B. performed research; A.J.B. analyzed data; A.J.B., M.H.D., and R.P.C. wrote the paper.

This work was supported by the Medical Research Council (Doctoral Training Award Studentship to A.J.B., Grant MC-A060-5PQ70 to R.P.C., and Grant MC-A060-5PQ80 to M.H.D.). A.J.B. is currently affiliated with the Ear Institute, University College London, United Kingdom, and thanks Timothy D. Griffiths and Ingrid S. Johnsrude for continuing financial support while he wrote this paper.

The authors declare no competing financial interests.

Correspondence should be addressed to Alexander J. Billig, UCL Ear Institute, 332 Gray's Inn Road, London WC1X 8EE, UK. E-mail: ajbillig@gmail.com.
}

intensity, or spatial location, the more likely they are to be integrated and represented as a single perceptual object or stream (van Noorden, 1975; Moore and Gockel, 2012). The processes that underlie integration and segregation are affected not only by these stimulus features, but also by internal states of the listener such as the degree to which they are attending to the sounds (Carlyon et al., 2001; Sussman et al., 2002; Snyder et al., 2006; Billig and Carlyon, 2016) and whether the stimuli correspond to a familiar speaker (Johnsrude et al., 2013) or word (Billig et al., 2013). The extent to which observers can voluntarily influence how they perceptually organize the outside world is unclear and

This is an open-access article distributed under the terms of the Creative Commons Attribution License Creative Commons Attribution 4.0 International, which permits unrestricted use, distribution and reproduction in any medium provided that the original work is properly attributed. 
A

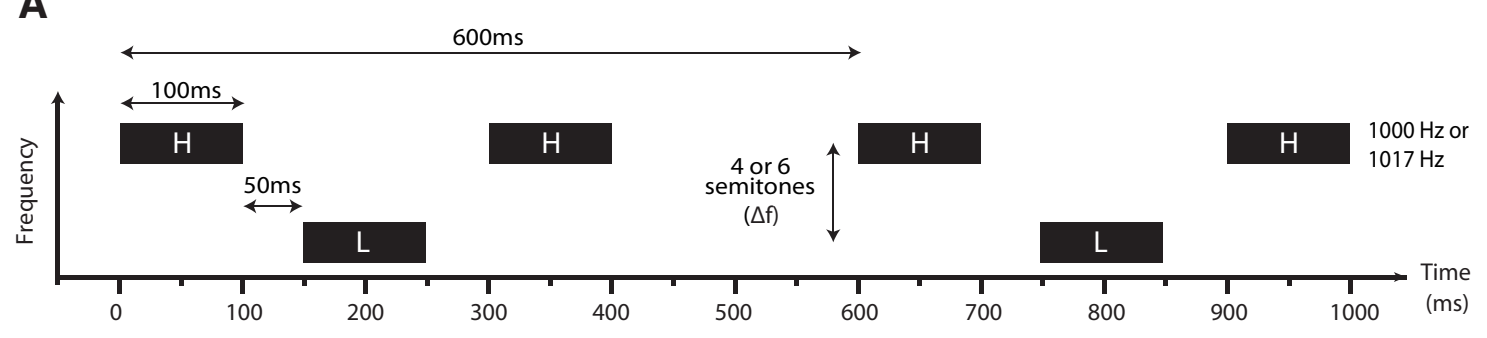

B

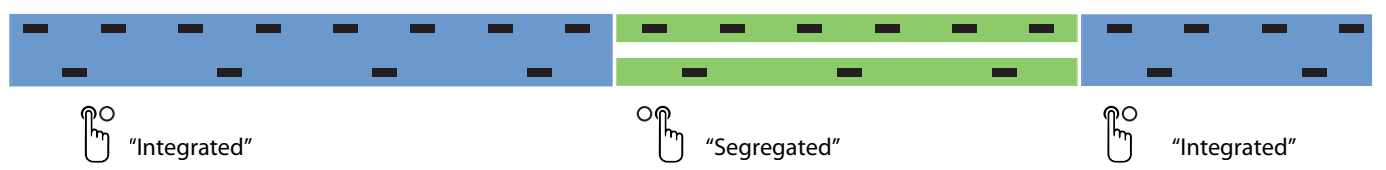

Figure 1. Stimulus parameters and percept reporting. $A$, Two triplets of a stimulus sequence consisting of $H$ and $L$ tones with a frequency separation $(\Delta f)$ of 4 or 6 semitones. The $H$ tone frequency was $1000 \mathrm{~Hz}$ for sound booth testing and $1017 \mathrm{~Hz}$ for testing with EEG/MEG. B, Illustrative changes in perceptual organization with corresponding button press reports. During integration (blue), $\mathrm{H}$ and $\mathrm{L}$ tones are perceived as belonging to a single pattern, whereas during segregation (green), they form two separate perceptual streams. Perception typically alternates every few seconds after a longer initial integrated phase.

bears on questions of whether and how higher-level cognition can influence perception (Fodor, 1983; Pylyshyn, 1999; Firestone and Scholl, 2016; Gross, 2017; Lupyan, 2017).

A common stimulus for investigating auditory perceptual organization is a repeating pattern of pure tones of high $(\mathrm{H})$ and low (L) frequencies, such as that shown in Figure $1 A$. For lower frequency separations and presentation rates, the sounds tend to be heard as integrated in a single stream that forms a distinctive galloping rhythm. At greater frequency separations and presentation rates, the $\mathrm{H}$ and $\mathrm{L}$ tones typically form two segregated streams (van Noorden, 1975). For a range of stimulus parameters, perception can alternate between the two percepts every few seconds, usually after a longer initial integrated phase (Carlyon et al., 2001; Pressnitzer and Hupé, 2006; Denham et al., 2013; Fig. 1B).

For such ambiguous sequences, listeners report being able to exert a degree of control over hearing integration or segregation (van Noorden, 1975; Pressnitzer and Hupé, 2006; Micheyl and Oxenham, 2010; Farkas et al., 2016). However, subjective responses may be affected by post-perceptual processes and biases such as shifts in decision criteria (Green and Swets, 1966) and attempts to meet the perceived aims of the experiment (Orne, 1962). To the extent that they vary with a listener's percept, indirect behavioral or neural measures can bypass such issues. For example, several EEG/MEG studies have detected more positive auditory cortical responses $\sim 60-100 \mathrm{~ms}$ after the onset of the middle tone in such triplets during reports of segregation compared with integration (Gutschalk et al., 2005; Hill et al., 2012; Szalárdy et al., 2013a). We argue that these objective neural measures can also shed light on the neural stages of processing that underlie any genuine effect of intention on perception.

Here, we combine subjective and objective measures to demonstrate an effect of intention on perception reflected in evoked responses in auditory cortex. To do so we measure neural activity with EEG/MEG as participants listen neutrally to HLH- sequences (Fig. 1A) and report spontaneous changes in their perception (Fig. 1B). We derive a univariate marker of perceptual organization in the auditory evoked field at the group level, but also make use of multiple temporal features in the neural response to train multivariate percept classifiers for each participant. We then study the relative occurrence of these neural signatures when participants actively try to promote integration (by attending to the whole pattern) or segregation (by attending exclusively to tones of one frequency). This allows us to establish whether their reports of successfully influencing their percept are supported by and reflected in stimulus-locked activity in auditory cortex or are instead more likely to have a post-perceptual locus.

\section{Materials and Methods}

\section{Participants}

Data were collected in two separate experimental settings. Twenty-five participants took part in a sound booth (setting 1), for the purposes of ensuring that stimulus parameters gave rise to integrated and segregated percepts in approximately equal measure and screening participants before EEG/MEG recording to ensure that they could experience both percepts. Twenty-two of these participants also took part in the EEG/MEG laboratory (setting 2) between 1 and $34 \mathrm{~d}$ later. Two further participants took part in setting 2 only after screening with an online test. All 27 participants across both settings were aged $18-40$ (mean $=28.56$ years, 12 females), right-handed, and reported no neurological or developmental disorders. They were recruited from the Medical Research Council Cognition and Brain Sciences Unit participant panel or by word of mouth and were paid for their time. One participant, whose results were not qualitatively different from the remainder of the group, had a threshold of $30 \mathrm{~dB}$ HL at $1500 \mathrm{~Hz}$ in the left ear. All other participants had normal hearing $(<25 \mathrm{~dB} H \mathrm{HL}$ pure tone thresholds over the range of the stimuli, 1000-2000 Hz). All experimental procedures were approved by the Cambridge Psychology Research Ethics Committee.

\section{Stimuli}

Sequences of $250 \mathrm{HLH}$-triplets were presented diotically, where $\mathrm{H}$ and L were $100 \mathrm{~ms}$ pure tones (Fig. $1 A$ ). The frequency of the $\mathrm{H}$ tone was fixed at $1000 \mathrm{~Hz}$ (setting 1) or $1017 \mathrm{~Hz}$ (setting 2), except for the final $\mathrm{H}$ tone in the final triplet, when it was $250 \mathrm{~Hz}$. The choice of $1017 \mathrm{~Hz}$ in setting 2 was to avoid possible contamination by harmonics of the $50 \mathrm{~Hz}$ line noise. The $250 \mathrm{~Hz}$ tone was low enough in frequency to be detectable on a low-pass filtered auxiliary channel of the MEG recording setup in setting 2 and was included to enable neural recordings to be time-locked to the stimulus. The frequency of the $\mathrm{L}$ tone in a given sequence was lower than that of the $\mathrm{H}$ tone by an amount $(\Delta \mathrm{f})$ of either four or six semitones (both settings). Silent intervals of $50 \mathrm{~ms}$ separated tones within a triplet and silent intervals of $200 \mathrm{~ms}$ separated one triplet from the next, giving sequences of $150 \mathrm{~s}$ duration. These stimuli were chosen to match Experiment 2 of Gutschalk et al. (2005).

Filler stimuli lasting a total of $40 \mathrm{~s}$ were created to separate experimental sequences from each other. These consisted of $5005 \mathrm{~ms}$ silence, fol- 
lowed by a $100 \mathrm{~ms} 250 \mathrm{~Hz}$ tone (a time-locking signal, with the same purpose as that described in the previous paragraph). This was followed by $1900 \mathrm{~ms}$ of silence, then by 33 pure tones, each of $100 \mathrm{~ms}$ duration with $50 \mathrm{~ms}$ of silence between tones. The frequencies of these tones were selected at random from a log-rectangular distribution from 200 to 2000 $\mathrm{Hz}$. Their purpose was to interfere with memory of the previous sequence in an effort to minimize context effects such as those described by Snyder et al. (2009). The filler stimulus continued with $22,945 \mathrm{~ms}$ of silence, another $100 \mathrm{~ms} 250 \mathrm{~Hz}$ tone (to warn the participant that the next experimental sequence was about to begin), and a final $5000 \mathrm{~ms}$ of silence. All tones in the experimental sequences and filler stimuli included $10 \mathrm{~ms}$ linear onset and offset ramps and were generated digitally at a sample rate of $44100 \mathrm{~Hz}$ with 16-bit resolution.

\section{Experimental procedures}

In setting 1, participants were seated in a double-walled sound-insulated room and sounds were presented over Sennheiser HD650 headphones at a level of $55 \mathrm{~dB}$ SPL. In setting 2, participants sat under the dewar of a VectorView system (Elekta Neuromag) while MEG and EEG activity was recorded (see "EEG and MEG acquisition and preprocessing" section for details of preparation and recording). In this setting, sounds were presented through tube headphones with silicone inserts at $50 \mathrm{~dB}$ above the participant's $1000 \mathrm{~Hz}$ pure tone hearing threshold. Using their right hand, participants pressed one computer key (setting 1) or button box button (setting 2) when hearing an integrated, galloping triplet pattern and another when hearing the tones segregate into two isochronous sequences (Fig. 1B). The screen indicated their most recent response that corresponded to their current percept. They were told to make a selection as soon as possible after the sequence began and to make further responses whenever their percept changed. There were four conditions with different instructions. In "neutral" sequences, participants were instructed to let their perception take a natural course. In "attempt integration" sequences, they tried to promote the integrated percept by attending to the whole pattern. In "attempt segregation" sequences, they tried to promote the segregated percept by attending either to the $\mathrm{H}$ tones ("attend high") or the L tones ("attend low").

The experiment consisted of two (setting 1) or four (setting 2) blocks. Each block contained five sequences: two neutral, one attempt integration, one attend high, and one attend low. In setting 1, the order of instruction conditions was the same in both blocks for a given participant; in setting 2, this order was reversed for the final two blocks. The two neutral sequences in a block never occurred consecutively and $\Delta \mathrm{f}$ alternated between four and six semitones from sequence to sequence. An onscreen message specified the instruction before and throughout each trial. Response key/button mapping and order of instruction and $\Delta \mathrm{f}$ conditions were balanced across participants. Participants relaxed between sequences and took breaks of at least a minute between blocks (while remaining under the dewar in setting 2). During experimental trials in setting 2 , they were instructed to keep their eyes open and to maintain fixation on a cross in the center of the screen, or elsewhere if more comfortable, to minimize alpha power and artifacts from eye movements. In setting 2, participants' head positions were checked at the start of each block and their position adjusted (to minimize loss of MEG signal) if they had dropped by $1 \mathrm{~cm}$ or more. Testing lasted $\sim 30 \mathrm{~min}$ in setting 1 and $\sim 60 \mathrm{~min}$ in setting 2 .

Before the experiment, the concept of streaming was explained using HLH- patterns with $\Delta \mathrm{f}$ of 0,5 , and 12 semitones. Participants practiced reporting their percept while listening neutrally. They were then told that they may be able to influence their percept by attending either to the whole pattern or to one or other sets of tones; these conditions were also practiced. Participants were told that it was far more important to be honest and accurate in their responses than to be successful in their attempts to influence their percept. In setting 2, practice and experimental blocks occurred after electrode preparation and head position digitization (described in the "Materials and Methods: EEG and MEG acquisition and preprocessing" section). The two participants who had not taken part in setting 1 completed an online training session to familiarize themselves with the stimuli and percept reporting process and to practice trying to influence their percept. Instructions were repeated in person immediately before the experiment. Those participants who had taken part in setting 1 more than 1 week previously also completed the online training as a refresher.

\section{EEG and MEG acquisition and preprocessing}

Magnetic fields were recorded using a VectorView system (Elekta Neuromag) with one magnetometer and two orthogonal planar gradiometers at each of 102 locations. Electric potentials were recorded concurrently using $70 \mathrm{Ag}-\mathrm{AgCl}$ sensors arranged in the extended $10-10 \%$ configuration fitted to the scalp using an electrode cap (Easycap) and referenced to an electrode on the nose with a ground electrode on the right cheek. Head position was monitored continuously using five head position indicator (HPI) coils. ECG and horizontal and vertical EOG activity was recorded with three pairs of electrodes. The positions of the EEG sensors, HPI coils, and $\sim 100$ additional head points were digitized with a 3D digitizer (Fastrak Polhemus) relative to three anatomical fiducial points (the nasion and both preauricular points). Data were acquired with a sampling rate of $1000 \mathrm{~Hz}$ and a high-pass filter of $0.01 \mathrm{~Hz}$. For the magnetometer and gradiometer recordings, the temporal extension of Signal Space Separation in MaxFilter was used to identify bad channels, suppress noise sources, and compensate for head movement. For all sensor types, additional noisy channels were identified and excluded for each participant based on observations during recording and offline visual inspection, as were recording segments containing SQUID jumps, channel pops, and muscle activity. Line noise at $50 \mathrm{~Hz}$ and its harmonics was removed using adaptive multitaper regression implemented in the EEGLAB (Delorme and Makeig, 2004; RRID:SCR_007292) plugin CleanLine, after which all activity was downsampled to $250 \mathrm{~Hz}$. Independent components analysis (ICA) was performed in EEGLAB using the Infomax routine (with subGaussian components included) on a version of the data that had been high-pass filtered at $0.5 \mathrm{~Hz}$ ( $6 \mathrm{~dB}$ cutoff, $1 \mathrm{~Hz}$ transition band, FIR windowed sinc filter) to impose the stationarity assumed by ICA. EEG channels were considerably noisier than magnetometers and gradiometers and did not improve the quality of the decomposition. They were therefore discarded and subsequent analyses were restricted to magnetometers and gradiometers only. Components corresponding to eye blinks/ movements and cardiac artifacts were identified and projected out of another copy of the data that had been low-pass filtered at $30 \mathrm{~Hz}(6 \mathrm{~dB}$ cutoff, $6.667 \mathrm{~Hz}$ transition band, FIR windowed sinc filter) and high-pass filtered at $0.278 \mathrm{~Hz}$ ( $6 \mathrm{~dB}$ cutoff, $0.556 \mathrm{~Hz}$ transition band, FIR windowed sinc filter). This high-pass filter was selected for reasons explained in the next paragraph.

The resulting data were divided into $600 \mathrm{~ms}$ epochs, each beginning at the start of an HLH- triplet. Epochs beginning $<1500 \mathrm{~ms}$ after a button press (or the start of the sequence) or ending $<1500 \mathrm{~ms}$ before a button press (or the end of the sequence) were excluded from analyses. This minimized neural and muscular activity related to movement and removed periods around transitions when the reported percept was least likely to be reliable. Baseline correction was not performed due to the repeating nature of the stimulus precluding a sufficient silent period between triplets, which meant that neural responses from one epoch were likely to carry over to the next. Due to the exclusion of epochs close to reported perceptual switches, any such influence should arise solely from triplets with the same (reported) perceptual state, and epoch time can therefore be thought of as circular (for a similar approach, see Hill et al., 2012). The high-pass filter of $0.278 \mathrm{~Hz}$ corresponds to a $3600 \mathrm{~ms}$ time period, the shortest possible interval between retained epochs corresponding to different perceptual reports. The relatively conservative approach of epoch rejection necessary for tapping periods that were as perceptually stable as possible led to a median retention rate of $58 \%$ (2900 epochs) per participant, which is comparable to that in Hill et al. (2012).

\section{Dipole fitting}

Pairs of equivalent current dipoles were fitted to the magnetometer and gradiometer data for each participant separately, using the VB-ECD approach in SPM12 (v6685; RRID:SCR_007037). Reconstructions made use of single shell forward models based on participant-specific T1weighted structural MRI scans. Sensor positions were projected onto 
each participant's MRI by minimizing the sum of squared differences between the digitized fiducials and MRI scan fiducials and between the digitized head shape and the individual scalp mesh. The VB-ECD routine uses a variational Bayes approach to iteratively optimize location and orientation parameters of fitted dipoles. The midpoints of each hemisphere's Heschl's gyrus were used as soft location priors with no priors for dipole orientation. Fitting was performed separately for magnetometer and gradiometer data using the mean activity in the $24 \mathrm{~ms}$ window centered on the first prominent turning point in the sensor space waveform (peaking 40-110 ms after triplet onset) over all epochs. The dipole pair that accounted for the most variance in sensor data out of 20 iterations of the fitting process was selected for each participant and each sensor type. Using these dipoles as spatial filters, further analyses were conducted on the hemisphere-specific source waveforms and on the mean waveform across hemispheres. Because the polarity of reconstructed waveforms depends on the orientation of the sources with respect to individual anatomy, each participant's source waveforms were inspected and inverted as necessary such that the first prominent turning point (peaking 40-110 ms after triplet onset) was a local maximum. All results were comparable across magnetometers and gradiometers and are reported for gradiometers only. Fitted dipole pairs accounted for a mean of $91.9 \%$ (SD $4.2 \%$ ) of the variance in the sensor recordings over the fitting window and were located in or close to Heschl's gyrus for all hemispheres (mean MNI coordinates [ $\pm 49-213$ ], SD $6 \mathrm{~mm}$ ).

To verify that our findings were not dependent on the use of location priors in Heschl's gyrus, we performed a separate set of analyses, selecting for each participant the neural component from the ICA that had the maximum back-projected power in the evoked response. Dipoles fitted to these components also had a mean location in Heschl's gyrus and the reconstructed source waveforms showed qualitatively similar results to those described below. Although, for some participants, these reconstructed sources were located in regions remote from auditory cortex, their locations were not consistent across participants and were not considered further.

\section{Experimental design and statistical analysis}

Sample size justification. No published research has used the same approach to test for intention effects with the same stimuli; however, effect sizes for two relevant findings can be estimated from previous studies: (1) an intention effect on behavioral streaming measures of $\eta^{2}=0.70$ (Pressnitzer and Hupé, 2006) and (2) a percept effect on MEG evoked responses of $\eta^{2}=0.43$ (Gutschalk et al., 2005). Of the two effects, the latter would require the largest sample size to detect, namely 18 participants for $90 \%$ power. We tested 24 participants in the MEG setting, which allowed for dropouts and accounted for possible overestimation of effect sizes due to unreported null findings.

Behavioral analyses. Because there were no significant differences in the mean percentage of segregation reported across the sound booth and EEG/MEG laboratory settings $\left(t_{(15)}=1.38, p=0.189, d=0.23,95 \%\right.$ confidence interval [CI] [ $-0.200 .04]$, tested on the 16 participants with no conditions in either setting in which the percentage of segregation was 0 or 100), behavioral data were combined across the two settings. Of the 27 participants, two (both tested only in setting 1 ) were excluded from behavioral data analyses. Both had at least one $\Delta \mathrm{f} \times$ instruction condition with no sequences that met the following criteria: (1) the first reported phase was integrated and (2) at least two completed subsequent phases were reported. These criteria were necessary to allow separate analysis and comparison of the duration of initial-integrated, subsequent-integrated, and segregated phases. Percentages of segregation for all remaining participants were logit-transformed and phase durations were log-transformed before being submitted to repeated-measures ANOVAs for analysis as a function of $\Delta \mathrm{f}$ and instruction. These transformations typically produced data with normally distributed residuals. When this was not the case, nonparametric tests were also conducted; these gave rise to the same qualitative pattern of results and are not reported separately. Mean percentages/durations were calculated on the transformed scale and then converted back to percentages/seconds for reporting. Null hypothesis significance testing was applied with an alpha value of 0.05 . Degrees of freedom were adjusted for asphericity as appropriate using the Huynh-Feldt correction (uncorrected degrees of freedom are reported for clarity).

Univariate neural analyses. Epochs in the neutral condition were averaged for each combination of $\Delta \mathrm{f}$ and reported percept for each participant. To maximize power, epochs occurring before the first percept report were labeled as integrated and the first integrated phase of a sequence was not considered separately from the remaining integrated phases. The exclusion of any epochs before the first segregated report (consistent with some researchers' suggestions to treat these separately; Denham et al., 2013) led to qualitatively similar results. One participant, who had only five valid epochs in one $\Delta \mathrm{f} \times$ percept cell in the neutral condition, was excluded from subsequent analyses of neural data. All other participants had at least 55 valid epochs per $\Delta \mathrm{f} \times$ percept cell in the neutral condition (the mean across participants of number of epochs in smallest cell was 145). To assess neural activity as a function of percept without stimulus confounds, the time courses of the two $\Delta \mathrm{f}$ conditions were averaged within each percept before statistical analysis. Percept differences were similarly partialled out of analyses of neural activity as a function of $\Delta \mathrm{f}$.

Statistical differences between percepts and frequency separations in the neutral condition were assessed using a cluster-based permutation method (Maris and Oostenveld, 2007). Within-participants $t$ tests were conducted at each time point and the largest contiguous cluster of values all exceeding a critical $t$-value (corresponding to an alpha value of 0.05 ) was selected for further analysis. Cluster significance was assessed by comparison with a null distribution generated by randomly permuting the labels of condition averages 1000 times within each participant and using an alpha value of 0.05 .

Epochs in the attempt integration, attempt segregation (attend high), and attempt segregation (attend low) conditions were averaged within each $\Delta \mathrm{f}$ without regard to reported percept. Differences over the temporal cluster of interest from the neutral condition were derived for each of the following contrasts: (1) $1 / 2^{*}$ attempt segregation (attend high) $+1 / 2 *$ attempt segregation (attend low) - attempt integration; (2) attempt segregation (attend high) - attempt integration; (3) attempt segregation (attend low) - attempt integration; and (4) attempt segregation (attend high) - attempt segregation (attend low). In all cases, the two $\Delta$ f conditions were given equal weight. Paired $t$ tests were conducted on these differences.

To test whether effects of intention on univariate neural responses in the non-neutral conditions were as large as would be expected based on perceptual reports and under the assumption that the neural signature of percept in the neutral condition also applied in the non-neutral conditions, the following calculations were made. The percentages of each percept reported in each non-neutral condition for each $\Delta \mathrm{f}$ and participant were applied to the relevant mean neural response from the neutral condition. Simulated and observed values were compared using a paired $t$ test with an alpha value of 0.05 .

Multivariate neural analyses. Epochs were labeled and participants excluded as outlined in the "Experimental design and statistical analysis: univariate neural analyses" section. Support vector machines (SVMs) with linear kernels were trained to classify integrated versus segregated epochs in the neutral condition for each $\Delta \mathrm{f}$ and participant using an adapted version of the DDTBOX package (Bode et al., 2017; RRID: SCR_015978) in MATLAB (RRID:SCR_001622). To ensure that the classifiers were unbiased, random subsampling within each SVM was used to match the number of epochs across classes. Fivefold cross-validation was applied and the subsampling and cross-validation process was repeated 100 times. Features were the standardized values of the neural response at the 150 sampled time points of each $600 \mathrm{~ms}$ epoch (arising from the 250 $\mathrm{Hz}$ sampling frequency) and the cost parameter $(C)$ was set as 1 . Classifier performance in the neutral condition was assessed for each participant by comparing classified versus actual labels and averaging the percentage correct over the $5 \times 100=500$ iterations and over $\Delta$ f conditions. Group classification accuracy was tested against the $50 \%$ chance level using a $t$ test with an alpha value of 0.05 . Feature weights were obtained from the SVM training functions and corrected using the method of Haufe et al. (2014), which removes strongly weighted but theoretically irrelevant noise features. These were normalized across par- 
A

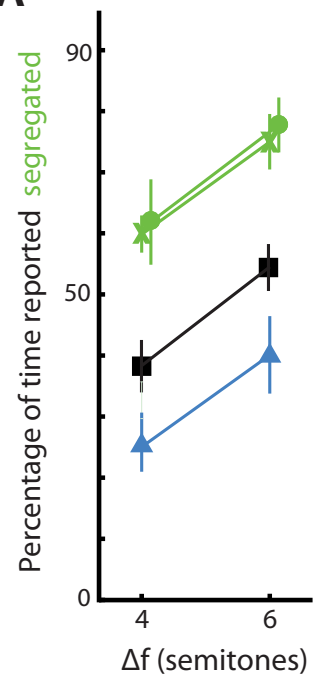

B

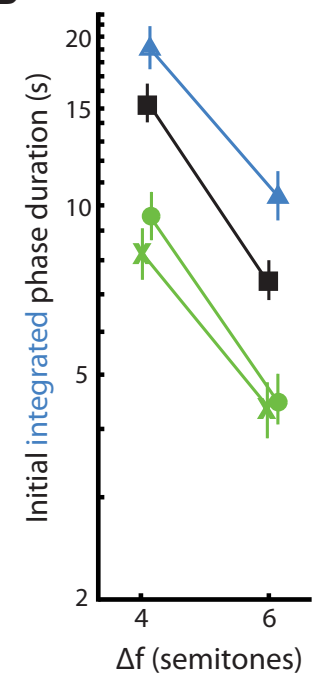

C

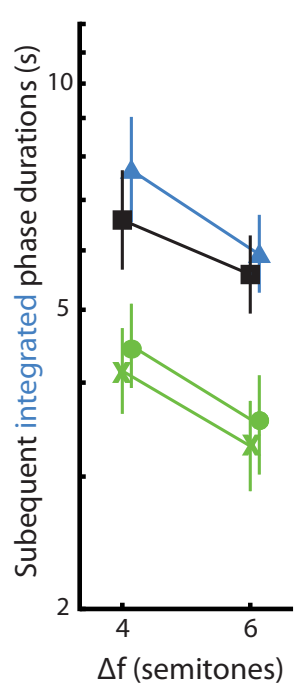

D

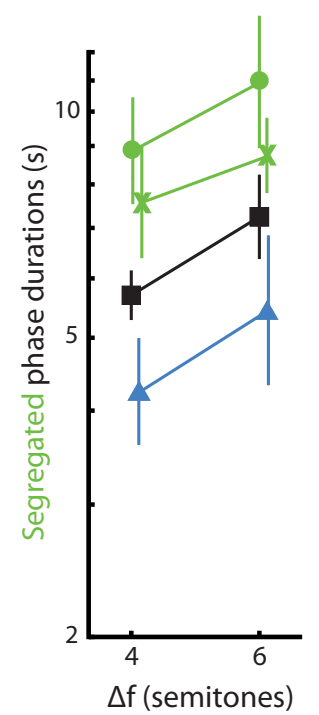

Listening instruction:

Neutral

Attempt Integration

X Attempt Segregation (Attend High)

Attempt Segregation (Attend Low)

Figure 2. Behavioral analyses. Shown are the effects of frequency separation $(\Delta f)$ and task on the percentage of time reporting segregation $(\boldsymbol{A})$, the duration of initial integrated phases $(\boldsymbol{B})$, the duration of subsequent integrated phases $(\boldsymbol{C})$, and the duration of segregated phases (D). Phase durations are plotted on a log scale. Black squares indicate listen neutrally; blue triangles, attempt integration; green crosses, attempt segregation by attending to high tones; and green circles, attempt segregation by attending to low tones. Error bars indicate within-participants $95 \%$ Cls.

Table 1. Statistics for effects of intention on phase durations (compared with the neutral condition)

\begin{tabular}{|c|c|c|c|c|c|}
\hline Task & Phases & $t$-value & $p$-value & $d$ & $d 95 \% \mathrm{Cl}$ \\
\hline \multirow[t]{3}{*}{ Attempt integration } & Initial integrated & 3.75 & 0.001 & 0.40 & {$[0.150 .65]$} \\
\hline & Subsequent integrated & 1.75 & 0.094 & 0.17 & {$[-0.030 .37]$} \\
\hline & Segregated & 3.90 & $<0.001$ & 0.56 & {$[0.220 .90]$} \\
\hline \multirow[t]{3}{*}{ Attempt segregation (attend high) } & Initial integrated & 5.30 & $<0.001$ & 0.70 & {$[0.361 .05]$} \\
\hline & Subsequent integrated & 4.95 & $<0.001$ & 0.85 & {$[0.421 .28]$} \\
\hline & Segregated & 2.62 & 0.015 & 0.35 & {$\left[\begin{array}{lll}0.05 & 0.64\end{array}\right]$} \\
\hline \multirow[t]{3}{*}{ Attempt segregation (attend low) } & Initial integrated & 3.70 & 0.001 & 0.63 & {$\left[\begin{array}{lll}0.23 & 1.03\end{array}\right]$} \\
\hline & Subsequent integrated & 4.13 & $<0.001$ & 0.66 & {$[0.271 .04]$} \\
\hline & Segregated & 3.67 & 0.001 & 0.66 & {$\left[\begin{array}{lll}0.24 & 1.08\end{array}\right]$} \\
\hline
\end{tabular}

ticipants then averaged over $\Delta \mathrm{f}$ for plotting. The 500 trained SVMs for each $\Delta \mathrm{f}$ and participant were also used to classify all epochs in the nonneutral conditions regardless of percept report. The percentage classified as segregated was compared across non-neutral conditions using withinparticipant $t$ tests with an alpha value of 0.05 for the same contrasts as outlined in the "Univariate neural analyses" section.

To test whether task-related differences in the percentage of epochs classified as segregated was as high as would be expected based on subjective reports, it was necessary to take into account the accuracy of the trained classifiers in the neutral condition. The percentage of reports of segregation for each participant, frequency separation, and task was multiplied by (neutral classification accuracy -50$) / 50$ (i.e., the neutral classification accuracy above chance, as a proportion from -1 to 1 ). The expected task-related difference in the percentage of epochs classified as segregated was derived for each participant and frequency separation by taking the average of these adjusted percentages of segregated reports over the two attempt segregation conditions and subtracting the adjusted percentage of segregated reports in the attempt integration condition. These expected difference values were then averaged over frequency separations and compared with the observed differences using a paired $t$ test with an alpha value of 0.05 .

\section{Results}

\section{Behavioral results}

As shown in Figure 2A, segregation was reported for a greater proportion of time for the larger than for the smaller $\Delta \mathrm{f}$ for all tasks. This arose from a combination of shorter initial integrated phases (Fig. 2B), shorter subsequent integrated phases (Fig. 2C), and longer segregated phases (Fig. 2D). All of these effects were statistically significant (Fig. $2 A: F_{(1,24)}=34.89, p<0.001, \eta_{\mathrm{p}}^{2}=.59$, $95 \%$ CI [0.48 0.72]; Fig. $2 B: F_{(1,24)}=46.58, p<0.001, \eta_{\mathrm{p}}^{2}=.66,95 \%$ CI $\left[0.58\right.$ 0.77]; Figure 2C: $F_{(1,24)}=15.28, p<0.001$, $\eta_{\mathrm{p}}^{2}=.39,95 \%$ CI $[0.180 .58]$; and Figure $2 D: F_{(1,24)}=11.28, p<$ $0.001, \eta_{\mathrm{p}}^{2}=.32,95 \%$ CI [0.09 0.58]).

Importantly, the percentage of time each percept was reported was also affected by the task instructions $\left(F_{(1,24)}=\right.$ 51.55, $p<0.001, \eta_{\mathrm{p}}^{2}=0.68,95 \%$ CI [0.58 0.80]; Fig. $\left.2 A\right)$. This effect was reflected in extended phases of the intended percept (although not to a significant extent for non-initial integrated phases) and shortened phases of the unintended percept compared with the neutral condition (Fig. $2 B-D$; see Table 1 for statistics). Focusing on tones of a single frequency to promote segregation had a larger effect on the percentage of time hearing segregation than trying to hold the three tones in a triplet together (the black lines are closer to the blue lines than to the green lines in Fig. $2 A ; t_{(24)}=3.03, p=0.006, d=0.67,95 \% \mathrm{CI}$ $\left.\left[\begin{array}{ll}0.17 & 1.17\end{array}\right]\right)$. However, there was no effect of attending to the high versus the low tones during segregated listening $\left(t_{(24)}=\right.$ $1.29, p=0.208, d=0.27,95 \%$ CI $[-0.150 .69]$ ). 
A

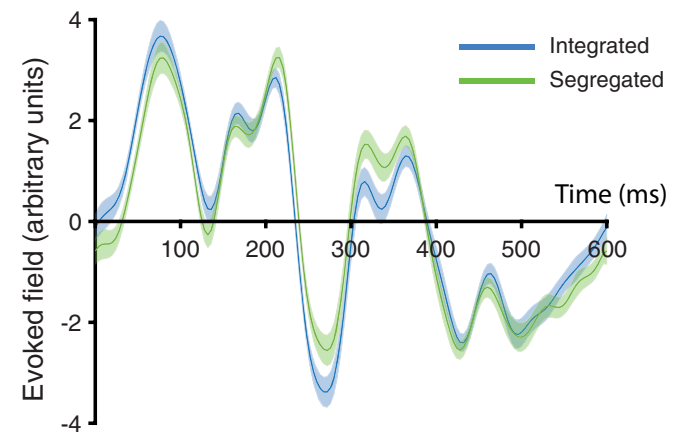

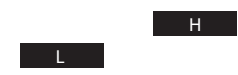

C

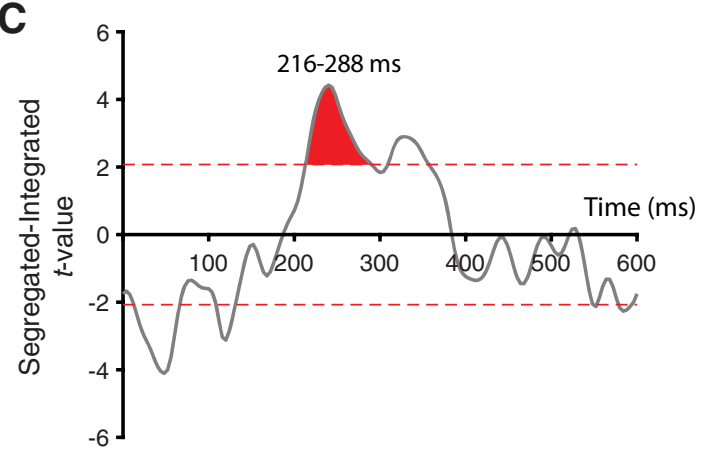

B

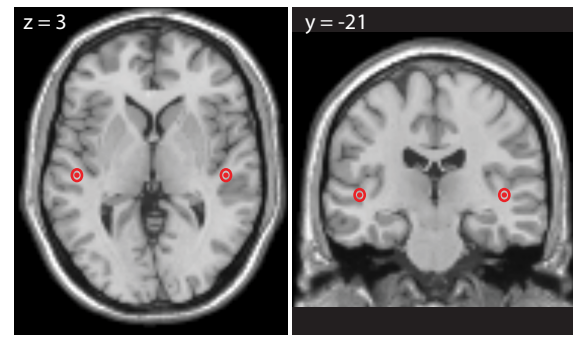

D

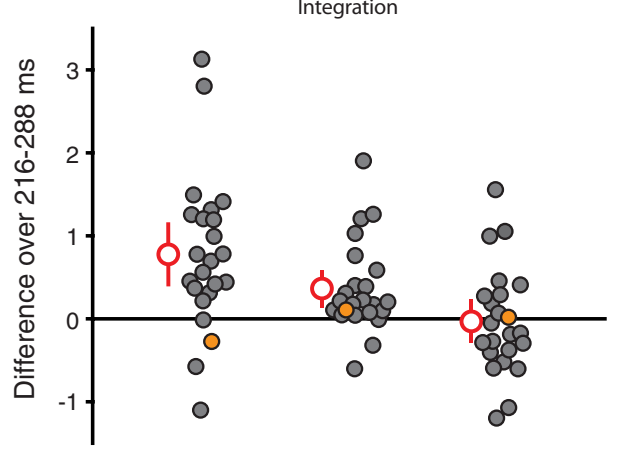

Figure 3. Univariate neural analyses. $A$, Group time course (mean and within-participants $95 \% \mathrm{Cls}$ ) of neural activity for integrated (blue) and segregated (green) reports in the neutral condition across frequency separations. Activity is projected through a spatial filter based on dipoles in bilateral auditory cortex fitted to the sensor data separately for each participant. The timing of each tone in the triplet is indicated below the plot. $\boldsymbol{B}$, Mean and within-participants $95 \% \mathrm{Cl}$ of the fitted dipole locations for the activity in $\boldsymbol{A}$. Sources are shown on a template brain with coordinates in MNI space. Mean reconstructed sources lie in bilateral posteromedial Heschl's gyrus. $C, t$-values for the neutral segregated minus integrated group difference wave across frequency separations. Dashed red lines indicate the critical $t$-values at $p=0.05$ and the shaded red area represents the largest suprathreshold cluster. $\boldsymbol{D}$, Differences in neural activity averaged over the time window of interest for neutral segregated minus integrated (left), attempt segregation minus attempt integration (middle), and attend high minus attend low (right), across frequency separations. Filled circles correspond to individual participants, with mean and $95 \%$ Cls shown in red. The orange circle represents a single participant also highlighted in Figure $4 B-D$ for comparison of results across univariate and multivariate approaches.

\section{Univariate neural results}

Neural responses time-locked to the onset of each HLH- triplet were extracted for each percept in the neutral condition independent of $\Delta \mathrm{f}$ (Fig. $3 A$ ), averaging over the dipoles in bilateral auditory cortices (Fig. $3 B$ ). A univariate analysis revealed a time window $216-288 \mathrm{~ms}$ posttriplet onset $(66-138 \mathrm{~ms}$ post $\mathrm{L}$ tone onset) during which epochs reported as segregated evoked a significantly more positive response than those reported as integrated independent of $\Delta \mathrm{f}$ (cluster-based permutation test, $p=$ .001 ; window-specific test, $t_{(22)}=4.18, p<0.001, d=0.32,95 \%$ CI [0.12 0.52]; Fig. $3 C$ ). When based on single dipoles, the size of the percept effect in this time window did not differ between the left and right hemispheres $\left(t_{(22)}=1.55, p=0.135, d=0.35,95 \%\right.$ CI $\left[\begin{array}{lll}-0.13 & 0.83\end{array}\right]$ ).

The effect of intention on the neural response in this window was determined by subtracting the mean over all epochs during attempts at integration from the mean over all epochs during attempts at segregation regardless of reported percept. The group difference was significantly greater than zero $\left(t_{(22)}=3.14, p=\right.$ $0.005, d=0.17,95 \%$ CI [0.05 0.29]; Fig. 3D, middle), paralleling the percept comparison in the neutral condition (Fig. 3D, left) and supporting participants' reports that they heard more segregation when they tried to do so than when they tried to hear integration. This effect is unlikely to be driven by attentionrelated modulations of neural responses to particular tones inde- pendent of perceptual organization; the conditions in which attention was focused on the $\mathrm{H}$ or the $\mathrm{L}$ tones did not differ significantly from each other $\left(t_{(22)}=0.23, p=0.819, d=0.01\right.$, 95\% CI [ $-0.110 .14]$; Fig. 3D, right). Importantly, there was also no evidence for a residual response bias; the magnitude of the neural difference in the non-neutral conditions was similar to that expected if all reports in those conditions were accurate $\left(t_{(22)}=0.38, p=0.710, d=0.08,95 \%\right.$ CI $\left.[-0.350 .51]\right)$.

\section{Multivariate neural results}

The difference waveform in the neutral condition (Fig. $3 C$ ) indicated that multiple time windows might be informative in distinguishing between integrated and segregated percepts beyond the 216-288 ms window determined from the univariate analysis. To make use of information across the entire epoch, we sought multivariate temporal patterns that distinguished between integrated and segregated percepts at a single-trial level and were allowed to vary across participants. Linear SVMs trained for each $\Delta \mathrm{f}$ and participant (Fig. $4 A$ ) achieved classification accuracy significantly above chance $\left(t_{(22)}=6.11, p<0.001, d=1.77,95 \% \mathrm{CI}\right.$ [1.17 2.86]; Fig. 4C); this was driven by responses in multiple time windows, including that identified in the univariate analysis (Fig. $4 B)$. When based on single dipoles, classifier performance did not differ between the left and right hemispheres $\left(t_{(22)}=1.02, p=\right.$ $0.321, d=0.22,95 \%$ CI $\left.\left[\begin{array}{lll}-0.21 & 0.68\end{array}\right]\right)$. 
The SVMs trained on neutral epochs were then used to classify epochs in the other conditions. Paralleling the univariate results, a greater percentage of epochs were classified as segregated when participants attempted segregation than when they tried to integrate the sounds $\left(t_{(22)}=\right.$ 3.87, $p<0.001, d=1.12,95 \%$ CI [0.63 1.77]; Fig. $4 D$, left). Again, this was not driven by epochs in which tones of one particular frequency were attended; the percentage of epochs classified as segregated was similar whether participants attended to high or low tones $t_{(22)}=0.45$, $p=0.657, d=0.13,95 \%$ CI [ $-0.590 .63]$; Fig. $4 D$, right).

The task-related difference in the percentage of epochs classified as segregated (mean 2.5\%) was more than an order of magnitude smaller than the difference in reported proportions (mean 36.6\%). This discrepancy was due to nonperfect classifier performance; although accuracy was above chance $(50 \%)$, the mean was only $53.2 \%$ and the maximum across participants $59.1 \%$. After taking into account the accuracy of each classifier, the task-related difference in the percentage of epochs classified as segregated was no different from that expected if all percept reports in the non-neutral conditions were accurate $\left(t_{(22)}=0.55, p=0.586, d=0.08,95 \% \mathrm{CI}\right.$ $\left.\left[\begin{array}{ll}-0.24 & 0.42\end{array}\right]\right)$. Consistent with the univariate analysis, there was therefore no evidence for a residual response bias.

The effect of intention determined by the multivariate analysis was larger and more reliable than that from the univariate analysis. The more flexible approach was able to exploit the data of participants whose neural activity did not align with the group percept signature in the 216288 ms time window. For example, one participant's percept in the neutral condition could be decoded above chance based on the activity at a range of time points, including an effect in the opposite direction from that of the group at $\sim 216 \mathrm{~ms}$ after triplet onset (Fig. $4 B$, dashed orange trace; Fig. $4 C$, orange circle).

\section{Discussion}

Our findings demonstrate that listeners can exert intentional control over how many objects they perceive in an ambiguous auditory scene. Differences in auditory cortical responses during attempts to hear repeating patterns of pure tones as an integrated whole versus segregated streams were consistent with signatures of these percepts obtained during a neutral listening condition. These differences supported listeners'
A
Classifier is trained on Neutral epochs reported as Integrated or Segregated

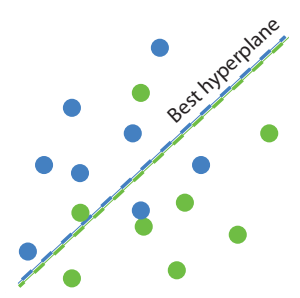

Classifier is applied to Attempt Segregation epochs applied to Attempt Integration epochs

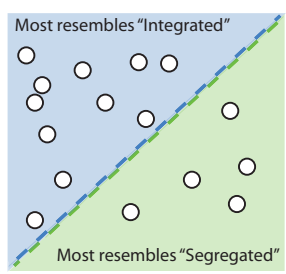

B

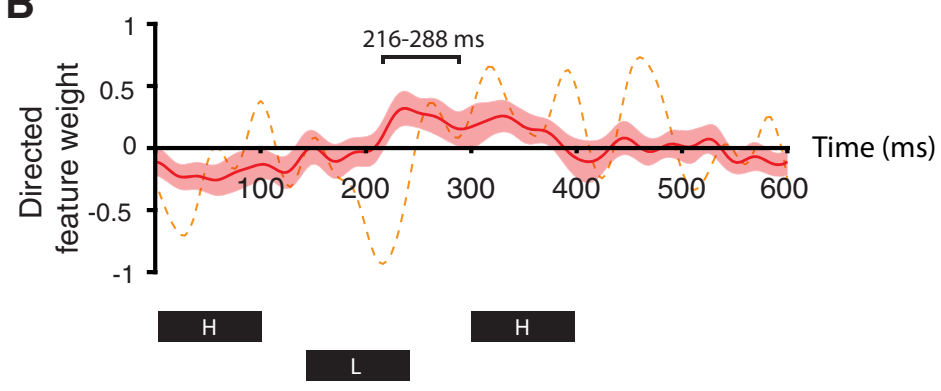

C

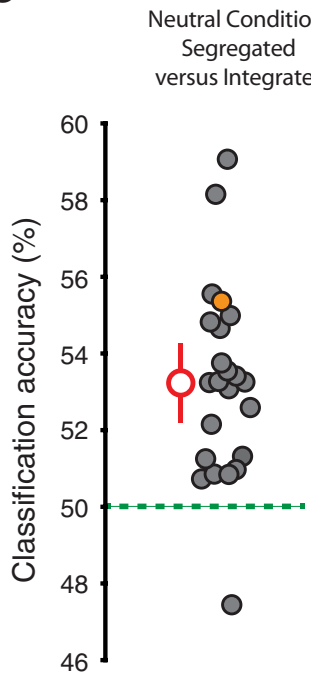

D

$\begin{array}{cc}\text { Attempt } & \text { Attend High } \\ \text { Segregation } & \text { - Attend Low } \\ \text { - Attempt } & \\ \text { Integration } & \end{array}$

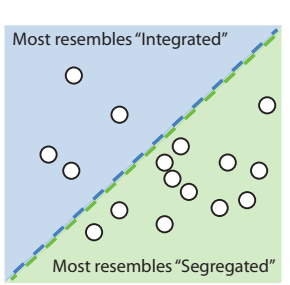

Most resembles "Segregated" 
subjective reports that they could, to some extent, "hear what they want to hear."

\section{Indexing low-level perception}

We argue that the activity measured during neutral listening relates to the percept rather than to decisions made during the process of reporting it. The inherent uncertainty of localization based on MEG precludes ascribing a primary versus nonprimary auditory cortical locus; our source reconstruction appears consistent with either of these. However, it seems unlikely that post-perceptual decision-related activity would originate from auditory regions and be so consistently timed from the onset of each stimulus. Furthermore, we excluded epochs surrounding button presses to minimize the contribution of activity relating to motor planning or execution. We therefore take the neutral neural signature to reflect perceptual experience. The use of bistable stimuli to probe perception also avoided acoustic confounds. Although we presented stimuli with two different frequency separations, leading to different reported proportions of segregation (cf. Gutschalk et al., 2005), the key comparisons of neural activity were between alternative percepts of identical sounds.

Our interpretation of activity in the non-neutral conditions assumes that the neural response carried more information about perception than about the instructions themselves, which differed in terms of how listeners were to attend to the sounds. Selective attention is known to affect the evoked response to tones even when perceptual organization is stable (Hillyard et al., 1973; Näätänen et al., 1978). Such modulations would presumably be maximally different across the two subconditions in which participants attended exclusively to either the $\mathrm{H}$ or $\mathrm{L}$ tones rather than between one of these subconditions and the case when listeners attended to all of the tones. However, we found no difference for attention to the $\mathrm{H}$ versus the $\mathrm{L}$ tones over the time window of interest in the univariate analysis or in the percentage of epochs classified as segregated in the multivariate analysis. We therefore argue that attention alone (without concomitant changes in perceptual organization) cannot account for the observed neural effects.

Another important feature of our design was the simultaneous collection of percept reports and neural data, allowing us to draw direct associations between the two. Some previous studies have inferred integration or segregation using measures sensitive to stimulus manipulations that also affect perceptual organization, such as the mismatch negativity (Sussman et al., 1999; Winkler et al., 2006; Carlyon et al., 2010) or performance on a deviant detection task (Carlyon et al., 2010; Micheyl and Oxenham, 2010; Billig et al., 2013; Spielmann et al., 2014). However, such measures are influenced by additional factors (Divenyi and Danner, 1977; Spielmann et al., 2013, 2014; Szalárdy et al., 2013b; Sussman et al., 2014) and the degree to which they, in isolation, can provide a reliable indication of perceptual organization over the course of sustained bistable stimulation is unclear.

\section{Implications for auditory scene analysis}

The more positive response for segregation compared with integration from 66-138 ms after the onset of the $\mathrm{L}$ tone was consistent with previous findings (Gutschalk et al., 2005; Hill et al., 2012; Szalárdy et al., 2013a). It may in part reflect an increased $\mathrm{P} 1 \mathrm{~m}$ response to the $\mathrm{L}$ tone during segregation due to a release from adaptation by responses to the previous $\mathrm{H}$ tone as neuronal receptive fields narrow and segregation occurs (Fishman et al., 2001; Gutschalk and Dykstra, 2014). However, our results do not depend on this interpretation; given the continuous stimulation paradigm, it is not clear how the observed differences relate to responses to individual tones. Furthermore, our participantspecific classification analysis indicated that this time window was not the most diagnostic of percept for all individuals. Variability across listeners may arise from distinct listening strategies or it may reflect differences in how multiple components from repeated sounds summate to an aggregate measured signal. Multivariate techniques such as representational similarity analysis have provided insight into the fine spatial patterns representing stimulus information in the brain (Haxby et al., 2001; Kriegeskorte et al., 2008). Here, we applied a different form of multivariate analysis, classification in the temporal domain, to reveal individualized percept-specific patterns in neural activity (see also Reichert et al., 2014; Wilbertz et al., 2017 for classification of bistable visual perception).

We observed effects of percept and intention when analyzing responses generated by neural sources in bilateral auditory cortex. fMRI has also revealed greater responses in precuneus and right intraparietal sulcus during segregation compared with integration (Cusack, 2005; Hill et al., 2011). Our analysis of precisely stimulus-locked responses would have been insensitive to more temporally diffuse effects that such studies may have tapped. Further evidence for the involvement in streaming of a network beyond auditory cortex comes from activity during perceptual reversals (as opposed to during stable periods of integration or segregation) in inferior colliculus, thalamus, insula, supramarginal gyrus, and cerebellum (Kondo and Kashino, 2009; Schadwinkel and Gutschalk, 2011; Kashino and Kondo, 2012). How these regions support or reflect either spontaneous reversals or voluntary switches remains to be established.

A distinction has been drawn between primitive and schemabased processes of perceptual organization (Bregman, 1990). Primitive processes automatically partition a scene based on its physical properties, whereas schema-based processes select elements based on attention or prior knowledge. One might expect different neural instantiations of the outcomes of these processes; however, we found the same segregation signature regardless of whether listeners allowed their perception to take a natural course, deliberately attended to the $\mathrm{H}$ tones, or deliberately attended to the $\mathrm{L}$ tones. We argue that the neural realization of an auditory scene may not only consist of distinct representations of attended and unattended streams of differing fidelity (Mesgarani and Chang, 2012; Puvvada and Simon, 2017), but also mark whether any segregation has occurred at all (Szalárdy et al., 2013b; cf. Gandras et al., 2017).

We asked participants to try to influence their percept by attending either to a subset of the tones or to all of them. The former approach may succeed by narrowing receptive fields of auditory cortical neurons such that different populations respond to the tones of each frequency (Fritz et al., 2007; Ahveninen et al., 2011) or by introducing a perceived loudness difference between H and L tones (van Noorden, 1975; Dai et al., 1991). In contrast, repeatedly shifting attention across frequencies may promote integration by disrupting these effects. The size of the change in reports from the neutral to the attempt segregation condition was greater than that from the neutral to the attempt integration condition. This was not the case in previous studies (Pressnitzer and Hupé, 2006; Micheyl and Oxenham, 2010), a fact that may reflect differences in stimuli or in how instructions were interpreted. We also note that, in our experiment, volitional control similarly affected reported durations of intended and unintended phases, whereas Pressnitzer and Hupé (2006) found that phases of unwanted percepts were curtailed to a greater de- 
gree than target phases were extended. Listeners in that study may have used additional strategies to shorten segregated phases, such as briefly diverting attention away from the tone sequence (Carlyon et al., 2003). Phase duration distributions have informed modeling of auditory scene analysis (Mill et al., 2013; Rankin et al., 2015) and prompted parallels to be drawn between different forms of bistability across sensory modalities (Pressnitzer and Hupé, 2006). We emphasize that the interaction between stimulus characteristics and high-level factors such as attention, which may differ across bistable phenomena, must be considered in general accounts of how the brain handles perceptual ambiguity (van Ee et al., 2005; Kogo et al., 2015).

\section{Summary}

Auditory bistability offers a powerful means of understanding how cognitive states such as listening goals, attention, and prior knowledge influence perception while controlling for stimulus differences. Linking subjective reports with neural measures on a trial-by-trial basis allows us to tap into low-level processes as opposed to post-perceptual decisions. This method identifies signatures of perceptual experience in auditory cortex to demonstrate that listeners can use attention not only to enhance the representation of a subset of sounds, but also to intentionally alter the number of distinct objects heard to make up the auditory scene.

\section{References}

Ahveninen J, Hämäläinen M, Jääskeläinen IP, Ahlfors SP, Huang S, Lin FH, Raij T, Sams M, Vasios CE, Belliveau JW (2011) Attention-driven auditory cortex short-term plasticity helps segregate relevant sounds from noise. Proc Natl Acad Sci U S A 108:4182-4187. CrossRef Medline

Billig AJ, Carlyon RP (2016) Automaticity and primacy of auditory streaming: concurrent subjective and objective measures. J Exp Psychol Hum Percept Perform 42:339-353. Medline

Billig AJ, Davis MH, Deeks JM, Monstrey J, Carlyon RP (2013) Lexical influences on auditory streaming. Curr Biol 23:1585-1589. CrossRef Medline

Bode S, Feuerriegel D, Bennett D, Alday PM (2017) The Decision Decoding ToolBOX (DDTBOX): a multivariate pattern analysis toolbox for eventrelated potentials. CrossRef

Bregman AS (1990) Auditory scene analysis: the perceptual organization of sound. Cambridge, MA: MIT.

Carlyon RP, Cusack R, Foxton JM, Robertson IH (2001) Effects of attention and unilateral neglect on auditory stream segregation. J Exp Psychol Hum Percept Perform 27:115-127. CrossRef Medline

Carlyon RP, Plack CJ, Fantini DA, Cusack R (2003) Cross-modal and nonsensory influences on auditory streaming. Perception 32:1393-1402. CrossRef Medline

Carlyon RP, Thompson SK, Heinrich A, Pulvermüller F, Davis MH, Shtyrov Y, Cusack R, Johnsrude IS (2010) Objective measures of auditory scene analysis. In: The neurophysiological bases of auditory perception (LopezPoveda EA, Palmer AR, Meddis R, eds), pp 507-519. New York, NY: Springer.

Cusack R (2005) The intraparietal sulcus and perceptual organization. J Cogn Neurosci 17:641-651. CrossRef Medline

Dai HP, Scharf B, Buus S (1991) Effective attenuation of signals in noise under focused attention. J Acoust Soc Am 89:2837-2842. CrossRef Medline

Delorme A, Makeig S (2004) EEGLAB: an open source toolbox for analysis of single-trial EEG dynamics. J Neurosci Methods 134:9-21. CrossRef Medline

Denham SL, Gyimesi K, Stefanics G, Winkler I (2013) Perceptual bistability in auditory streaming: how much do stimulus features matter? Learn Percept 5:73-100. CrossRef

Divenyi PL, Danner WF (1977) Discrimination of time intervals marked by brief acoustic pulses of various intensities and spectra. Percept Psychophys 21:125-142. CrossRef

Farkas D, Denham SL, Bendixen A, Winkler I (2016) Assessing the validity of subjective reports in the auditory streaming paradigm. J Acoust Soc Am 139:1762-1772. CrossRef Medline

Firestone C, Scholl BJ (2016) Cognition does not affect perception: evaluating the evidence for "top-down" effects. Behav Brain Sci 39:e229. Medline

Fishman YI, Reser DH, Arezzo JC, Steinschneider M (2001) Neural correlates of auditory stream segregation in primary auditory cortex of the awake monkey. Hear Res 151:167-187. CrossRef Medline

Fodor JA (1983) The modularity of mind. Cambridge, MA: MIT.

Fritz JB, Elhilali M, David SV, Shamma SA (2007) Does attention play a role in dynamic receptive field adaptation to changing acoustic salience in $\mathrm{Al}$ ? Hear Res 229:186-203. CrossRef Medline

Gandras K, Grimm S, Bendixen A (2017) Electrophysiological correlates of speaker segregation and foreground-background selection in ambiguous listening situations. Neuroscience. In press. CrossRef Medline

Green DM, Swets JA (1966) Signal detection theory and psychophysics. New York, NY: Wiley.

Gross S (2017) Cognitive penetration and attention. Front Psychol 8:221. CrossRef Medline

Gutschalk A, Dykstra AR (2014) Functional imaging of auditory scene analysis. Hear Res 307:98-110. CrossRef Medline

Gutschalk A, Micheyl C, Melcher JR, Rupp A, Scherg M, Oxenham AJ (2005) Neuromagnetic correlates of streaming in human auditory cortex. J Neurosci 25:5382-5388. CrossRef Medline

Haufe S, Meinecke F, Görgen K, Dähne S, Haynes JD, Blankertz B, Bie $\beta$ mann $\mathrm{F}$ (2014) On the interpretation of weight vectors of linear models in multivariate neuroimaging. Neuroimage 87:96-110. CrossRef Medline

Haxby JV, Gobbini MI, Furey ML, Ishai A, Schouten JL, Pietrini P (2001) Distributed and overlapping representations of faces and objects in ventral temporal cortex. Science 293:2425-2430. CrossRef Medline

Hill KT, Bishop CW, Yadav D, Miller LM (2011) Pattern of BOLD signal in auditory cortex relates acoustic response to perceptual streaming. BMC Neurosci 12:85. CrossRef Medline

Hill KT, Bishop CW, Miller LM (2012) Auditory grouping mechanisms reflect a sound's relative position in a sequence. Front Hum Neurosci 6:158. CrossRef Medline

Hillyard SA, Hink RF, Schwent VL, Picton TW (1973) Electrical signs of selective attention in the human brain. Science 182:177-180. CrossRef Medline

Johnsrude IS, Mackey A, Hakyemez H, Alexander E, Trang HP, Carlyon RP (2013) Swinging at a cocktail party: voice familiarity aids speech perception in the presence of a competing voice. Psychol Sci 24:1995-2004. CrossRef Medline

Kashino M, Kondo HM (2012) Functional brain networks underlying perceptual switching: auditory streaming and verbal transformations. Philos Trans R Soc Lond B Biol Sci 367:977-987. CrossRef Medline

Kogo N, Hermans L, Stuer D, van Ee R, Wagemans J (2015) Temporal dynamics of different cases of bi-stable figure-ground perception. Vision Res 106:7-19. CrossRef Medline

Kondo HM, Kashino M (2009) Involvement of the thalamocortical loop in the spontaneous switching of percepts in auditory streaming. J Neurosci 29:12695-12701. CrossRef Medline

Kriegeskorte N, Mur M, Bandettini P (2008) Representational similarity analysis - connecting the branches of systems neuroscience. Front Syst Neurosci 2:4. CrossRef Medline

Lupyan G (2017) Changing what you see by changing what you know: the role of attention. Front Psychol 8:553. CrossRef Medline

Maris E, Oostenveld R (2007) Nonparametric statistical testing of EEG- and MEG-data. J Neurosci Methods 164:177-190. CrossRef Medline

Mesgarani N, Chang EF (2012) Selective cortical representation of attended speaker in multi-talker speech perception. Nature 485:233-236. CrossRef Medline

Micheyl C, Oxenham AJ (2010) Objective and subjective psychophysical measures of auditory stream integration and segregation. J Assoc Res Otolaryngol 11:709-724. CrossRef Medline

Mill RW, Bõhm TM, Bendixen A, Winkler I, Denham SL (2013) Modelling the emergence and dynamics of perceptual organisation in auditory streaming. PLoS Comput Biol 9:e1002925. CrossRef Medline

Moore BC, Gockel HE (2012) Properties of auditory stream formation. Philos Trans R Soc Lond B Biol Sci 367:919-931. CrossRef Medline

Näätänen R, Gaillard AW, Mäntysalo S (1978) Early selective-attention effect on evoked potential reinterpreted. Acta Psychol (Amst) 42:313-329. CrossRef Medline 
Orne MT (1962) On the social psychology of the psychological experiment: with particular reference to demand characteristics and their implications. Am Psychol 17:776-783. CrossRef

Pressnitzer D, Hupé JM (2006) Temporal dynamics of auditory and visual bistability reveal common principles of perceptual organization. Curr Biol 16:1351-1357. CrossRef Medline

Puvvada KC, Simon JZ (2017) Cortical representations of speech in a multitalker auditory scene. J Neurosci 37:9189-9196. CrossRef Medline

Pylyshyn Z (1999) Is vision continuous with cognition? The case for cognitive impenetrability of visual perception. Behav Brain Sci 22:341-365; discussion 366-423. Medline

Rankin J, Sussman E, Rinzel J (2015) Neuromechanistic model of auditory bistability. PLOS Comput Biol 11:e1004555. CrossRef Medline

Reichert C, Fendrich R, Bernarding J, Tempelmann C, Hinrichs H, Rieger JW (2014) Online tracking of the contents of conscious perception using real-time fMRI. Front Neurosci 8:116. CrossRef Medline

Schadwinkel S, Gutschalk A (2011) Transient bold activity locked to perceptual reversals of auditory streaming in human auditory cortex and inferior colliculus. J Neurophysiol 105:1977-1983. CrossRef Medline

Snyder JS, Alain C, Picton TW (2006) Effects of attention on neuroelectric correlates of auditory stream segregation. J Cogn Neurosci 18:1-13. CrossRef Medline

Snyder JS, Holder WT, Weintraub DM, Carter OL, Alain C (2009) Effects of prior stimulus and prior perception on neural correlates of auditory stream segregation. Psychophysiology 46:1208-1215. CrossRef Medline

Spielmann M, Schröger E, Kotz SA, Pechmann T, Bendixen A (2013) Using a staircase procedure for the objective measurement of auditory stream integration and segregation thresholds. Front Psychol 4:534. CrossRef Medline

Spielmann MI, Schröger E, Kotz SA, Bendixen A (2014) Attention effects on auditory scene analysis: insights from event-related brain potentials. Psychol Res 78:361-378. CrossRef Medline

Sussman E, Ritter W, Vaughan HG Jr (1999) An investigation of the auditory streaming effect using event-related brain potentials. Psychophysiology 36:22-34. CrossRef Medline

Sussman E, Winkler I, Huotilainen M, Ritter W, Näätänen R (2002) Topdown effects can modify the initially stimulus-driven auditory organization. Brain Res Cogn Brain Res 13:393-405. CrossRef Medline

Sussman ES, Chen S, Sussman-Fort J, Dinces E (2014) The five myths of MMN: redefining how to use MMN in basic and clinical research. Brain Topogr 27:553-564. CrossRef Medline

Szalárdy O, Bõhm TM, Bendixen A, Winkler I (2013a) Event-related potential correlates of sound organization: early sensory and late cognitive effects. Biol Psychol 93:97-104. CrossRef Medline

Szalárdy O, Winkler I, Schröger E, Widmann A, Bendixen A (2013b) Foreground-background discrimination indicated by event-related brain potentials in a new auditory multistability paradigm. Psychophysiology 50:1239-1250. CrossRef Medline

van Ee R, van Dam LCJ, Brouwer GJ (2005) Voluntary control and the dynamics of perceptual bi-stability. Vision Res 45:41-55. CrossRef Medline

van Noorden LPAS (1975) Temporal coherence in the perception of tone sequences. Eindhoven, The Netherlands: University of Technology.

Wilbertz G, van Kemenade BM, Schmack K, Sterzer P (2017) fMRI-based decoding of reward effects in binocular rivalry. Neuroscience of Consciousness 3:1-10.

Winkler I, van Zuijen TL, Sussman E, Horváth J, Näätänen R (2006) Object representation in the human auditory system. Eur J Neurosci 24:625-634. CrossRef Medline 